\section{Ocular pulse amplitude and retrobulbar blood flow change in dipper and non-dipper individuals}

${ }^{1}$ Yuzuncu Yil University, Medical School, Department of Ophthalmology, Van, Turkey

${ }^{2}$ Fatih University, Medical School, Department of Ophthalmology, Emek, Ankara, Turkey

${ }^{3}$ Fatih University, Medical School, Radiology

Department, Emek, Ankara, Turkey

${ }^{4}$ Fatih University, Medical School, Cardiology Department, Emek, Ankara, Turkey

${ }^{5}$ Fatih University, Medical School, Department of Internal Medicine, Section of Nephrology, Emek, Ankara, Turkey

Correspondence: R Karadag, Department of Ophthalmology, Yuzuncu Yil University, Medical School. Kazim Karabekir caddesi, Van 65200, Turkey Tel: + 90432 2262177; Fax: + 904322167519

E-mail: drrkaradag@ yahoo.com

Received: 2 December 2009 Accepted in revised form: 5 February 2011 Published online: 18 March 2011

\begin{abstract}
Purpose To evaluate ocular pulse amplitude (OPA), IOP values, and hemodynamic changes in the ophthalmic artery, central retinal artery, and short posterior ciliary artery in dipper and non-dipper patients.

Methods A total of 59 right eye measurements of healthy subjects with normotensive were included to the study. Ambulatory blood pressure (BP) monitoring measurement (ABPM), Doppler imaging, and OPA measurements were performed in the same day. The patients in which systolic BP decreased during the nocturnal time by $10 \%$ of the diurnal BP or more were called dippers. A patient whose nocturnal systolic BP fell by $<10 \%$ or even rose was defined as non-dipper. Color Doppler imaging was used for blood flow velocity assessment of ophthalmic, central retinal, and posterior ciliary arteries. For each artery, peak systolic and end-diastolic velocities (PSV and EDV, respectively), resistive index (RI), and pulsalite index (PI) were automatically calculated by the machine. Mean IOP and OPA values were calculated after three consecutive measurements. Results The mean OPA in non-dipper patients was significantly lower compared with that of dipper patients $(P=0.011)$. There was no significant difference in IOP levels between groups. There was no significant difference in the PSV, EDV, RI, and PI in the ophthalmic, posterior ciliary, and central retinal arteries between the groups. Conclusion Our study demonstrated that OPA level in non-dippers is lower than dippers. This may give additional information about the effect of BP changes on OPA values. Eye (2011) 25, 762-766; doi:10.1038/eye.2011.50; published online 18 March 2011
\end{abstract}

R Karadag', UC Keskin², A Koktener ${ }^{3}$, Y Selcoki ${ }^{4}$, IF Hepsen ${ }^{2}$ and M Kanbay ${ }^{5}$

Keywords: intraocular pressure; ocular pulse amplitude; retrobulbar blood flow; dipping-non-dipping blood pressure

\section{Introduction}

Several risk factors like hemodynamic changes, vascular dysregulation, and ocular blood flow decrease are thought to have a part in ocular diseases like glaucomatous optic nerve damage, ${ }^{1}$ diabetic retinopathy, ${ }^{2}$ and retinal vascular occlusive diseases. ${ }^{3}$

Hemodynamic changes like nocturnal hypotension were suggested to have a role in pathogenesis of glaucomatous optic nerve damage and anterior ischemic optic neuropathy. ${ }^{4}$ In some studies it has been shown that nocturnal blood pressure (BP) decrease is much more in glaucoma patients than normal patients. ${ }^{5-7}$ After correcting for age, intraocular pressure, and mean BP during retrobulbar blood flow measurements, a lower end-diastolic velocity and a higher resistivity index were found in the central retinal artery of glaucoma patients showing a decrease in mean BP during the night of more than $20 \%$ from the average daytime mean BP (over-dippers), when compared with glaucoma patients with a decrease not exceeding 20\% (dippers and nondippers). ${ }^{6}$ However, there are many questions still waiting answers in understanding the etiopathogenesis of glaucoma.

Circardian rhythm of BP can be measured by ambulatory BP monitoring. BP measurements without a fall in nocturnal BP are called non-dipping and with a fall in nocturnal $\mathrm{BP}$ is called dipping. ${ }^{8}$ Non-dipper patients with essential hypertension are prone to some diseases like left ventricular hypertrophy and 
stroke probably because of exposing to higher BP levels for 24 h. ${ }^{8,9}$ Excessive lowering of BP levels in dipping patients also increase risk for some diseases like cardiac ischemia and silent cerebrovascular damage. ${ }^{10-13}$

Color Doppler imaging is a non-invasive and reproducible imaging method. ${ }^{14}$ It has been used in occlusive and other diseases of eye in which vascular etiology was suspected as a causative factor..$^{15}$ Moreover, several color Doppler imaging studies have shown retrobulbar blood flow velocity reduction and resistivity index elevation in glaucoma patients. ${ }^{16-18}$

Ocular pulse amplitude is thought to be an indirect indicator of the choroidal perfusion and reflects the ocular blood flow corresponding to the heart pulse as a function of time. ${ }^{19}$ The relationship between ocular pulse amplitude (OPA) and hemodynamic changes was also investigated in some studies. ${ }^{20,21}$ OPA can be measured with dynamic contour tonometer, which is a nonapplation tonometer. It measures IOP levels and OPA without influence of anterior segment structures by a contour matched pressure sensing tip. ${ }^{22,23}$

To the best of our knowledge, the relationship between OPA obtained by DCT and dipper and non-dipper patients has not been studied. The purpose of this study is to evaluate OPA values and hemodynamic changes in the ophthalmic artery (OA), central retinal artery (CRA), and short posterior ciliary artery (PCA) in dipper and non-dipper patients. In the light of these previous studies, we speculate that non-dipping pattern in BP may also be a risk factor that causes target organ damage in the eye.

\section{Materials and methods}

This is a cross-sectional study. Normal healthy individuals and patients with normotensive were assessed in our department. Patients with history of hypertension or on any antihypertensive treatment were not included to the study. A full ophthalmic examination was achieved for each person, which included refraction, slit-lamp examination, and assessment of posterior segment. A total of 59 participants with no history of ocular disease, ocular surgery, ocular trauma, and any kind of anti-glaucomatic therapy were accepted to the study. The study was conducted according to the Helsinki Declaration and all of the patients were informed and the study was approved by the Local Ethics Committee of our hospital. Ambulatory BP monitoring (ABPM), Doppler imaging, and OPA measurement were performed in the same day.

The measurements of intraocular pressure and OPA were taken on the same day between 0800 and 1100 hours by the same ophthalmologist. Mean IOP and OPA values were calculated after three consecutive measurements. Dynamic contour tonometer displays the OPA value in $\mathrm{mmHg}$ and the quality score of the measurements, $\mathrm{Q}$, ranging from excellent, 1, to poor, 4 or 5 , were simultaneously displayed on the screen. The measurements with quality 1 and 2 were taken into account.

All participants underwent 24-h ABPM on a usual working day. They were told to work and act normally. The Tracker NIBP2 (Reynolds Medical Ltd, Hertford, UK) oscillometric monitor was used for ABPM. Each patient used an arm cuff of a similar size to the one used for routine office BP measurement in the non-dominant arm. The device measured BP for every $15 \mathrm{~min}$ between 0600 and 2300 hours, and every $30 \mathrm{~min}$ between 2300 and 0600 hours. The patients in which systolic BP decreased during the nocturnal time by $10 \%$ of the diurnal BP or more were called dippers. A patient whose nocturnal systolic BP fell by $<10 \%$ or even rose was defined as a non-dipper.

All the subjects underwent blood flow velocity assessment of their ophthalmic, central retinal, and posterior ciliary arteries by color Doppler imaging. Retrobulbar color Doppler US examination was performed with an imaging system (Sonoline Antares, Siemens, Erlangen, Germany) with a VF13-5 MHz, VX9-4 MHz multifrequency transducer by an experienced investigator (AK). Doppler examination was performed after a 20-min-rest between 0900 and 1200 hours. The transducer was applied to the closed eyelid using a coupling gel and avoiding any pressure to the globe. Patients were in supine position with a head tilted forward $\sim 30^{\circ}$ angle. After the gray-scale examination, the color Doppler imaging of the vessels was performed. Spectral waveforms and quantitative information were obtained using the minimum sized Doppler gate, appropriate Doppler frequency with an angle under $30^{\circ}$. A low to medium flow setting as chosen for slow flow (especially CRA and PCA), a medium to high flow setting was chosen in fast flow (especially for OA). CRA was found identified within the optic nerve, $2-4 \mathrm{~mm}$ posterior to the optic disc. OA was seen in the deeper part of the orbit, near the optic nerve medially $10-15 \mathrm{~mm}$ posterior to the globe. Temporal PCA was examined where surrounding the globe, near the optic nerve laterally. For each artery, peak systolic and end-diastolic velocities (PSV and EDV, respectively), resistive index (RI), and pulsalite index (PI) were automatically calculated by the machine. For each vessel, two consecutive measurements were obtained and the average value was taken. The entire examination of each patient lasted about $15 \mathrm{~min}$.

Measurements from the right eye were used for statistical analysis. Data were analyzed using parametric and non-parametric tests as appropriate using SPSS 
Table 1 Demographic data of the study groups

\begin{tabular}{lccc}
\hline & Dipper & Non-dipper & P-value \\
\hline Number of patients & 27 & 32 & \\
Gender (F/M) & $16 / 11$ & $23 / 9$ & 0.312 \\
Age (years, mean $\pm \mathrm{SD})$ & $51.4 \pm 12.0$ & $51.2 \pm 11.2$ & 0.976 \\
Systolic BP & $122.4 \pm 9.3$ & $125.8 \pm 10.8$ & 0.149 \\
(mm Hg, mean $\pm \mathrm{SD})$ & & & \\
Diastolic BP & $79.6 \pm 7.9$ & $82.3 \pm 9.6$ & 0.303 \\
(mm Hg, mean $\pm \mathrm{SD})$ & & & \\
IOP (mm Hg, mean $\pm \mathrm{SD})$ & $16.4 \pm 1.6$ & $16.5 \pm 1.8$ & 0.692 \\
OPA (mm Hg, mean $\pm \mathrm{SD})$ & $2.8 \pm 0.9$ & $2.3 \pm 0.6$ & 0.011 \\
\hline
\end{tabular}

Abbreviations: $\mathrm{BP}$, blood pressure; F, female; IOP, intraocular pressure $\mathrm{M}$, male; OPA, ocular pulse amplitude.

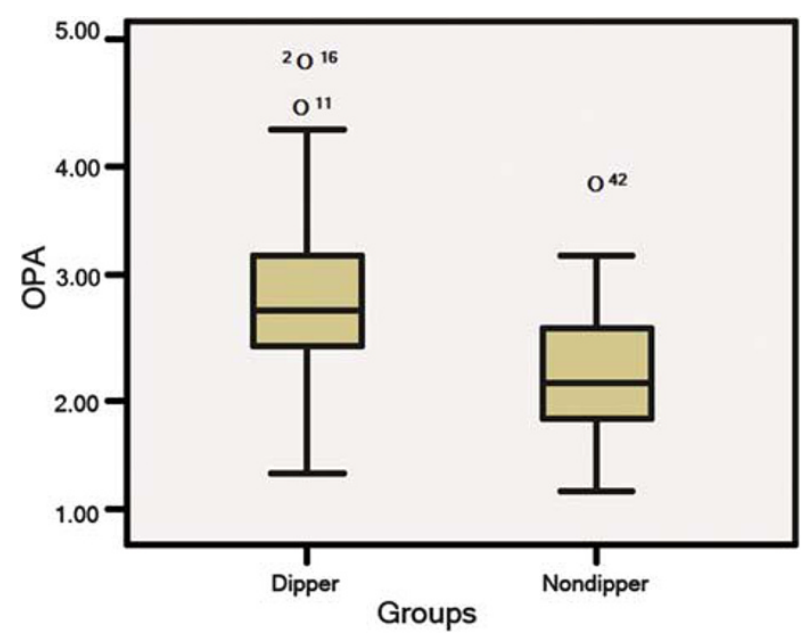

Figure 1 The distribution of OPA levels in both groups.

software 15.0 programs (SPSS, Chicago, IL, USA). Clinical characteristic were compared using $t$-test for parametric values and Mann-Whitney $U$-test for nonparametric values. The results of measurements were expressed as means \pm SD and were analyzed statistically via $P$-values of less than 0.05 that were considered to be statistically significant.

\section{Results}

The general characteristics of the subjects are summarized in Table 1 . There was no significant difference in the mean age $(P=0.976)$, mean BP $(P=0.149)$ for systolic BP and $(P=0.303)$ for diastolic BP, and IOP $(P=0.692)$ between the groups. In addition, the mean OPA in non-dipper patients was significantly lower compared with that of dipper patients $(P=0.011)$. Figure 1 shows the distribution of OPA levels in both groups. The retrobulbar blood flow velocities and resistivity indices of the subjects are represented in Table 2. There was no significant difference in the PSV,
Table 2 Hemodynamic values (median (min-max)) measured in the retrobulbar vessels for the study groups

\begin{tabular}{lccc}
\hline & Dipper & Non-dipper & P-value \\
\hline Ophthalmic artery & & & \\
PSV (cm/s) & $48.9(27.5-80.5)$ & $47.2(24.2-74.1)$ & 0.482 \\
EDV (cm/s) & $12.7(7.9-27.6)$ & $14.4(5.6-23.2)$ & 0.453 \\
RI & $0.7(0.6-0.9)$ & $0.7(0.6-0.9)$ & 0.180 \\
PI & $1.5(1.0-2.4)$ & $1.4(1.0-2.1)$ & 0.146 \\
& & & \\
Central retinal artery & & \\
PSV (cm/s) & $15.3(7.6-20.9)$ & $13.9(8.6-23.7)$ & 0.288 \\
EDV (cm/s) & $3.1(1.7-7.0)$ & $3.0(2.0-6.1)$ & 0.347 \\
RI & $0.8(0.6-0.9)$ & $0.8(0.6-0.9)$ & 0.462 \\
PI & $1.6(1.0-3.5)$ & $1.8(1.0-3.1)$ & 0.288 \\
& & & \\
Posterior ciliary artery & & \\
PSV (cm/s) & $17.7(9.6-27.2)$ & $16.2(7.5-29.3)$ & 0.491 \\
EDV (cm/s) & $3.6(2.3-9.1)$ & $3.1(2.3-14.2)$ & 0.081 \\
RI & $0.8(0.4-0.9)$ & $0.8(0.5-0.9)$ & 0.360 \\
PI & $1.6(0.7-2.3)$ & $1.7(0.8-2.5)$ & 0.235 \\
\hline
\end{tabular}

Abbreviations: EDV, end-diastolic velocity; max, maximum; min, minimum; PI, pulsatilite index; PSV, peak systolic velocity; RI, resistivity index. Statistical analysis was done using Mann-Whitney $U$-test.

EDV, RI, and PI in the ophthalmic $(P=0.482, P=0.453$, $P=0.180, P=0.146$, respectively) central retinal $(P=0.288, P=0.347, P=0.462, P=0.288$, respectively $)$ and posterior ciliary $(P=0.491, P=0.081, P=0.360$, $P=0.235$, respectively) arteries between the groups.

\section{Discussion}

The salient findings of the present study are as follows: (i) OPA levels were statistically significantly lower in non-dipper group compared with dipper group, (ii) there is not a significant difference in IOP, PSV, EDV, RI, and PI between dipper and non-dipper groups.

OPA values have been widely studied in the literature and it seems to be characteristic for different types of glaucoma and directly dependent on intraocular pressure levels. ${ }^{24}$ In primary open angle glaucoma and normotensive glaucoma patients, OPA values obtained with dynamic contour tonometry were found to be decreased $^{25}$ and increased OPA levels seemed to be correlated with less severe glaucomatous damage. ${ }^{26}$ In our study IOP levels in both groups were not significantly different but when OPA levels were significantly lower in non-dipper group.

The relationship between OPA obtained with DCT and BP changes had not been studied as widely as OPA and glaucoma relationship. Grieshaber $e t a^{20}$ studied the relationship between pulse amplitude and systemic BP parameters. They measured the BP and OPA values simultaneously in healthy subjects. They were reported that left ventricular ejection time was related but systolic 
and diastolic BPs were not related with OPA values. In another study, systolic pressure was positively correlated with OPA whereas diastolic pressure was negatively correlated with OPA but the BP measurements in this study were not performed continuously or concomitantly OPA measurements. ${ }^{27}$ Pourjavan et al ${ }^{28}$ investigated the physiologic diurnal variability of OPA. They did not find correlation between OPA and BP parameters but BP measurements were also not done simultaneously with OPA measurement. In our study, BP was measured continuously with ABPM and OPA was significantly lower in non-dipper patients.

There are various possible pathophysiologic mechanisms underlying impairment of the BP decline during nighttime. Extrinsic and intrinsic factors, including imbalance between the sympathetic nerve system and parasympathetic nerve system tone, particularly the failure to shift from sympathetic to parasympathetic during sleep, lack of physical activity, nutritional factors such as increased dietary sodium intake, and smoking, have all been studied in the genesis of blunted BP circadian rhythm. Certain diseases such as diabetes and chronic renal diseases may also strongly affect the (normal) circadian BP rhythm. ${ }^{29}$

Relationship between BP and IOP had been shown in previous studies. The study performed according to the data of Beaver Dam Eye Study showed that changes in IOP are directly and significantly associated with changes in systemic BPs. ${ }^{30}$ In an animal study with hypertensive rats positive relation was also found between BP and IOP. ${ }^{31}$ In our study there was no difference in IOP levels between non-dipper and dippers.

Optic nerve blood flow changes were thought to be an important factor in some ocular diseases like glaucoma. Grunwald et $a l^{32}$ studied the effect of systemic hypertension on glaucoma patients. Glaucoma patients without systemic hypertension showed lower optic nerve blood flow than patients with hypertension. They proposed that modulation of BP in glaucoma patients may affect the ocular blood flow. In studies about ocular blood flow in POAG and NPG, RI values were increased and EDV and PSV values were decreased. ${ }^{33-36}$ Gherghel et al studied the retrobulbar blood flow in glaucoma patients with nocturnal overdipping. RI was higher and EDV was lower in overdipping patients than non-dippers. These results suggest that patients with a marked nocturnal drop in systemic BP may harbor some kind of vascular dysregulation leading to alterations in the ocular circulation. ${ }^{6}$ In our study, we grouped the normal patients without glaucoma as dipper and nondipper and we found no significant difference in retrobulbar blood flow between groups.

In conclusion, our study demonstrated that OPA levels in non-dippers are lower than dippers and this may give additional information about the effect of BP changes on OPA values. We found no difference in ocular blood flow parameters between non-dipper and dipper patients. The possible etiologic reasons of BP decline during nighttime, as described before may be the cause of difference in OPA levels in non-dippers. Further studies are needed for finding the OPA changes in dipper/non-dippers especially in glaucoma patients and affect of these changes on glaucomatous optic nerve damage.

\section{Summary}

What was known before

- There is not a significant difference in IOP, PSV, EDV, RI, and PI between dipper and non-dipper groups.

What this study adds

- OPA levels were statistically significantly lower in non-dipper group compared with dipper group.

\section{Conflict of interest}

The authors declare no conflict of interest.

\section{References}

1 Flammer J, Orgül S, Costa VP, Orzalesi N, Krieglstein GK, Serra LM et al. The impact of ocular blood flow in glaucoma. Prog Retin Eye Res 2002; 21: 359-393.

2 Dimitrova G, Kato S, Tamaki Y, Yamashita H, Nagahara M, Sakurai $\mathrm{M}$ et al. Choroidal circulation in diabetic patients. Eye 2001; 15: 602-607.

3 Schicke SH, Duncker GI. Retinal color Doppler scanning of arteria centralis retinae by retinal diseases and healthy people. Klin Monatsbl Augenheilkd 2007; 224: 775-779.

4 Hayreh SS, Podhajsky P, Zimmerman MB. Role of nocturnal arterial hypotension in optic nerve head ischemic disorders. Ophthalmologica 1999; 213: 76-96.

5 Kaiser HJ, Flammer J. Systemic hypotension: a risk factor for glaucomatous damage? Ophthalmologica 1991; 203: 105-108.

6 Gherghel D, Orgül S, Gugleta K, Flammer J. Retrobulbar blood flow in glaucoma patients with nocturnal overdipping in systemic blood pressure. Am J Ophthalmol 2001; 132: 641-647.

7 Kaiser HJ, Flammer J, Graf T, Stümpfig D. Systemic blood pressure in glaucoma patients. Graefes Arch Clin Exp Ophthalmol 1993; 231: 677-680.

8 Verdecchia P, Schillaci G, Porcellati C. Dippers versus non-dippers. J Hypertens 1991; 9: 42-44.

9 Selcoki Y, Uz B, Baybek N, Akcay A, Eryonucu B. The effects of nocturnal dipping on cardiovascular outcomes and proteinuria in essential hypertensive patients. Cent Eur J Med 2008; 3: 279-286.

10 Pierdomenico SD, Bucci A, Costantini F, Lapenna D, Cuccurullo F, Mezzetti A. Circadian blood pressure changes and myocardial ischemia in hypertensive patients with coronary artery disease. J Am Coll Cardiol 1998; 31: 1627-1634.

11 Kurpesa M, Trzos E, Drozdz J, Bednarkiewicz Z, Krzemiń M. Myocardial ischemia and autonomic activity in dippers and 
non-dippers with coronary artery disease: assessment of normotensive and hypertensive patients. Int J Cardiol 2002; 83: 133-142.

12 Shimada K, Kario K. Altered circadian rhythm of blood pressure and cerebrovascular damage. Blood Press Monit 1997; 2: 333-338.

13 Kario K, Pickering TG, Matsuo T, Hoshide S, Schwartz JE, Shimada K. Stroke prognosis and abnormal nocturnal blood pressure falls in older hypertensives. Hypertension 2001; 38: 852-857.

14 Baxter GM, Williamson TH. Color Doppler imaging of the eye: normal ranges, reproducibility, and observer variation. J Ultrasound Med 1995; 14: 91-96.

15 Lieb WE. Color Doppler imaging of the eye and orbit. Radiol Clin North Am 1998; 36: 1059-1071.

16 Akarsu C, Bilgili MY. Color Doppler imaging in ocular hypertension and open-angle glaucoma. Graefes Arch Clin Exp Ophthalmol 2004; 242: 125-129.

17 Birinci H, Danaci M, Oge I, Erkan ND. Ocular blood flow in healthy and primary open-angle glaucomatous eyes. Ophthalmologica 2002; 216: 434-437.

18 Rankin SJ, Walman BE, Buckley AR, Drance SM. Color Doppler imaging and spectral analysis of the optic nerve vasculature in glaucoma. Am J Ophthalmol 1995; 119: 685-693.

19 Kaufmann C, Bachmann LM, Robert YC, Thiel MA. Ocular pulse amplitude in healthy subjects as measured by dynamic contour tonometry. Arch Ophthalmol 2006; 124: 1104-1108.

20 Grieshaber MC, Katamay R, Gugleta K, Kochkorov A, Flammer J, Orgül S. Relationship between ocular pulse amplitude and systemic blood pressure measurements. Acta Ophthalmol 2009; 87: 329-334.

21 Esgin H, Alimgil ML, Erda S. The effect of systemic hypertension on pulsatile ocular blood flow in diabetic patients. Acta Ophthalmol Scand 2001; 79: 160-162.

22 Kanngiesser HE, Kniestedt C, Robert YC. Dynamic contour tonometry: presentation of a new tonometer. J Glaucoma 2005; 14: 344-350.

23 Duba I, Wirthlin AC. Dynamic contour tonometry for post-LASIK intraocular pressure measurements. Klin Monatsbl Augenheilkd 2004; 221: 347-350.

24 Romppainen T, Kniestedt C, Bachmann LM, Stürmer J. Ocular pulse amplitude: a new biometrical parameter for the diagnose of glaucoma? Ophthalmologe 2007; 104: 230-235.
25 Stalmans I, Harris A, Vanbellinghen V, Zeyen T, Siesky B. Ocular pulse amplitude in normal tension and primary open angle glaucoma. J Glaucoma 2008; 17: 403-407.

26 Weizer JS, Asrani S, Stinnett SS, Herndon LW. The clinical utility of dynamic contour tonometry and ocular pulse amplitude. J Glaucoma 2007; 16: 700-703.

27 Detry-Morel M, Jamart J, Detry MB, Ledoux A, Pourjavan S. Clinical evaluation of the Pascal dynamic contour tonometer. J Fr Ophtalmol 2007; 30: 260-270.

28 Pourjavan S, Boëlle PY, Detry-Morel M, De Potter P. Physiological diurnal variability and characteristics of the ocular pulse amplitude (OPA) with the dynamic contour tonometer (DCT-Pascal). Int Ophthalmol 2007; 27: 357-360.

29 Kanbay M, Turgut F, Uyar ME, Akcay A, Covic A. Causes and mechanisms of nondipping hypertension. Clin Exp Hypertens 2008; 30: 585-597.

30 Klein BE, Klein R, Knudtson MD. Intraocular pressure and systemic blood pressure: longitudinal perspective: the Beaver Dam Eye Study. Br J Ophthalmol 2005; 89: 284-287.

31 Vaajanen A, Mervaala E, Oksala O, Vapaatalo H. Is there a relationship between blood pressure and intraocular pressure? An experimental study in hypertensive rats. Curr Eye Res 2008; 33: 325-332.

32 Grunwald JE, Piltz J, Hariprasad SM, Dupont J, Maguire MG. Optic nerve blood flow in glaucoma: effect of systemic hypertension. Am J Ophthalmol 1999; 127: 516-522.

33 Galassi F, Nuzzaci G, Sodi A, Casi P, Cappelli S, Vielmo A. Possible correlations of ocular blood flow parameters with intraocular pressure and visual-field alterations in glaucoma: a study by means of color Doppler imaging. Ophthalmologica 1994; 208: 304-308.

34 Galassi F, Nuzzaci G, Sodi A, Casi P, Vielmo A. Color Doppler imaging in evaluation of optic nerve blood supply in normal and glaucomatous subjects. Int Ophthalmol 1992; 16: $273-276$.

35 Harris A, Sergott RC, Spaeth GL, Katz JL, Shoemaker JA, Martin BJ. Color Doppler analysis of ocular vessel blood velocity in normal-tension glaucoma. Am J Ophthalmol 1994; 118: 642-649.

36 Rankin SJ, Walman BE, Buckley AR, Drance SM. Color Doppler imaging and spectral analysis of the optic nerve vasculature in glaucoma. Am J Ophthalmol 1995; 119: 685-693. 